\title{
Self-amplified spontaneous emission free electron laser devices and nonideal electron beam transport
}

\author{
L. L. Lazzarino, ${ }^{1}$ E. Di Palma, ${ }^{3}$ M. P. Anania, ${ }^{1}$ M. Artioli, ${ }^{2}$ A. Bacci, ${ }^{7}$ M. Bellaveglia, ${ }^{1}$ \\ E. Chiadroni, ${ }^{1}$ A. Cianchi, ${ }^{9}$ F. Ciocci, ${ }^{3}$ G. Dattoli, ${ }^{3}$ D. Di Giovenale,${ }^{1}$ G. Di Pirro, ${ }^{1}$ M. Ferrario, ${ }^{1}$ \\ G. Gatti, ${ }^{1}$ L. Giannessi, ${ }^{3,4}$ A. Mostacci, ${ }^{8}$ P. Musumeci, ${ }^{10}$ A. Petralia, ${ }^{3}$ V. Petrillo,${ }^{5,7}$ R. Pompili, ${ }^{1}$ \\ J. V. Rau, ${ }^{6}$ A. R. Rossi, ${ }^{7}$ E. Sabia, ${ }^{3}$ C. Vaccarezza, ${ }^{1}$ and F. Villa ${ }^{1}$ \\ ${ }^{1}$ INFN Laboratori Nazionali di Frascati, Via E. Fermi 45, 00044 Frascati, Rome, Italy \\ ${ }^{2}$ ENEA Centro Ricerche Bologna, via Martiri di Monte Sole 4, 40129 Bologna, Italy \\ ${ }^{3}$ ENEA Centro Ricerche Frascati, via E. Fermi 45, 00044 Frascati, Rome, Italy \\ ${ }^{4}$ Sincrotrone Trieste, 34149 Basovizza Trieste, Italy \\ ${ }^{5}$ Universitá degli Studi di Milano, via Celoria 16, 20133 Milano, Italy \\ ${ }^{6}$ Istituto di Struttura della Materia, ISM-CNR, Via del Fosso del Cavaliere, 100-00133 Rome, Italy \\ ${ }^{7}$ INFN-Mi, via Celoria 16, 20133 Milano, Italy \\ ${ }^{8}$ Rome University La Sapienza, Piazzale Aldo Moro 5, 00185 Roma, Italy \\ ${ }^{9}$ Universitá di Roma II Tor Vergata, Via della Ricerca Scientifica 1, 00133 Roma, Italy \\ ${ }^{10}$ UCLA, Los Angeles, California 90095, USA
}

(Received 29 January 2014; published 21 November 2014)

\begin{abstract}
We have developed, at the SPARC test facility, a procedure for a real time self-amplified spontaneous emission free electron laser (FEL) device performance control. We describe an actual FEL, including electron and optical beam transport, through a set of analytical formulas, allowing a fast and reliable on-line "simulation" of the experiment. The system is designed in such a way that the characteristics of the transport elements and the laser intensity are measured and adjusted, via a real time computation, during the experimental run, to obtain an on-line feedback of the laser performances. The detail of the procedure and the relevant experimental results are discussed.
\end{abstract}

DOI: 10.1103/PhysRevSTAB.17.110706

PACS numbers: 41.75.-i, 41.60.Cr, 41.85.Ja

\section{INTRODUCTION}

In a self-amplified spontaneous emission (SASE) free electron laser (FEL) the interaction of high quality electron beam with a long magnetic undulator provides the generation of bright EUV/x-ray pulses with suitable coherence properties for a different type of application [1-4]. The SASE FEL intensity growth and its associated details can be very well described in terms of numerical codes, which are designed to include as much physics as possible [5-9]. Even though highly reliable, these codes, accounting for almost all the aspects of actual devices, are rather slow in terms of simulation time. For this reason procedures $[10,11]$ employing analytical formulas became quite popular tools to design and/or to study FEL devices operating in either oscillator or SASE configurations.

The numerical codes are usually exploited to predict the behavior of a FEL device or for its design and optimization. Any FEL device requires an ad hoc treatment in terms of optimization, which is an ill posed concept, if expressed in abstract terms. It might be indeed convenient to provide the

Published by the American Physical Society under the terms of the Creative Commons Attribution 3.0 License. Further distribution of this work must maintain attribution to the author $(s)$ and the published article's title, journal citation, and DOI. conditions for the reduction of the saturation length by designing an appropriate transport channel or by using an external insertion like the so-called beam heater or even by studying solutions for the increase of the efficiency (for the different choices see e.g., [1-4]).

The study for the most convenient strategy is therefore not a straightforward task and the choice of designing the shorter saturation length may e.g., hamper the efficiency performances.

At the SPARC FEL [12] test facility a useful benchmarking tool has been developed to test the reliability of predictions given by different codes. Furthermore it could provide an important check of validation for models describing the nonlinear harmonic generation in SASE or seeded configuration [5-9]. Another important consideration emerging from this study is that semianalytical models are extremely reliable predicting tools, if properly used. They can accordingly be embodied within a suitable software environment to provide a kind of FEL virtual lab [13]. In this paper we describe the algorithm developed with its software implementation, starting from the theoretical model for the FEL evolution, and we show some experimental results suggested by the application of this tool.

The intensity growth along the undulator longitudinal coordinate $z$ is described by means of a generalized logistic map [11] [hereafter referred as SASE logistic map (SLAP)], namely, 


$$
P_{L}(z)=P_{0} \frac{A(z)}{1+\frac{P_{0}}{P_{F}} A(z)},
$$

where $P_{0}$ and $P_{F}$ are the seed and saturated power, $A(z)$ accounts for the small signal evolution, and the effect of saturation begins playing a role when $\frac{P_{0}}{P_{F}} A(z) \cong 1 . P_{0}$ and $A(z)$ depend on the specific operating conditions of the device. By assuming that the process occurs in pure SASE configuration we have $[10,11,14]$

$A(z)=\cosh \left(\frac{z}{L_{g}}\right)-\left(e^{-\frac{z}{2 L_{g}}} \cos \left(\frac{\pi}{3}-\frac{\sqrt{3} z}{2 L_{g}}\right)+e^{\frac{z}{2 L_{g}}} \cos \left(\frac{\pi}{3}+\frac{\sqrt{3} z}{2 L_{g}}\right)\right)$

with

$$
P_{0}[W] \propto \frac{186 \rho Q[C]}{(\lambda[m])^{2}}, \quad P_{F} \propto \rho P_{E},
$$

where $P_{E}$ denotes the e-beam power, $\rho$ is the Pierce parameter and $P_{0}$, associated with the bunch shot noise due to its charge $Q$, is given in practical units [15].

The key parameter in the previous equation is the gain length [11]

$$
L_{g}=\frac{\lambda_{u}}{4 \pi \sqrt{3} \rho}
$$

which in turns depends on the Pierce parameter $\rho$, whose $1 \mathrm{D}$ version is specified by [11]

$$
\rho=\frac{8.36 \times 10^{-3}}{\gamma}\left[J\left(\lambda_{u} K f_{b}\right)^{2}\right]^{1 / 3} .
$$

The above formula exhibits an explicit link with the relativistic energy factor $\gamma$, the undulator wave length $\lambda_{u}$ and strength parameter $K$, along with the Bessel factor contribution $f_{b}$.

The current density $J$, associated with the peak current $I$, and the e-beam transverse sections $\sigma_{\eta}(\eta=x, y)$, are given by

$$
J=\frac{I}{2 \pi \sigma_{x} \sigma_{y}}, \quad \sigma_{\eta}=\sqrt{\beta_{\eta} \epsilon_{\eta}}
$$

being $\beta_{\eta}$ and $\epsilon_{\eta} \quad(\eta=x, y)$ respectively the beta Twiss parameter and the beam emittance.

The SPARC focusing line, shown in Fig. 1, consists of six quadrupoles (namely the transfer line), driving the electron beam inside the undulator chain, composed by six permanent magnet sections and by five short quadrupoles, inserted within. The associated FODO lattice is realized by an undulator focusing in the vertical plane and by a quadrupole focusing in the other one.

The evolution of the $\beta_{\eta}$ Twiss parameters and its effects on the current density, as evident in Eq. (6), depend on the details of the electron beam transport along the line. It has already been stressed $[16,17]$ that an optimum strategy for such a design does not exist, since it is dependent on the quantity to be optimized. It might indeed happen that the conditions to

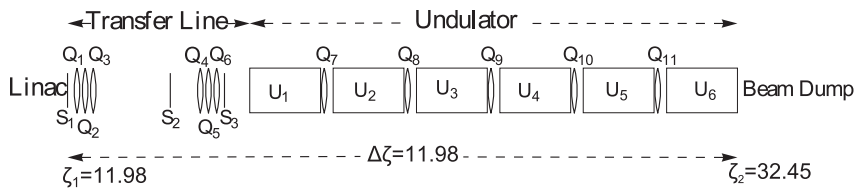

FIG. 1. Layout of the SPARC transfer line (quads $Q_{1} \rightarrow Q_{6}$ ) and undulator line (undulator sections $U_{1} \rightarrow U_{6}$ and quads $Q_{7} \rightarrow Q_{12}$ ). The $S$ 's tag the positions of the imaging screens, the $\zeta$ 's measure the distance from the photocathode.

enhance the output power are not those yielding the shortest saturation length [17]. Different strategies can be therefore developed and it is important that the transfer device can be flexible enough to allow real time variations while the machine is operating. Regarding the relevant design we make the distinction between two possible options, referred to as matching and transport respectively. By matching we mean that periodical conditions are imposed over the distance spanned by the single FODO unit (undulator module, short quadrupole and drifts between) as treated in detail in Ref. [18], while transport means that no specific constraints are imposed in setting the quadrupole currents if not an optimization of the FEL output intensity.

The behavior of the e-beam transverse size and the associated FEL performance as given by simulations are shown along the undulator respectively in Figs. 2 and 3 for
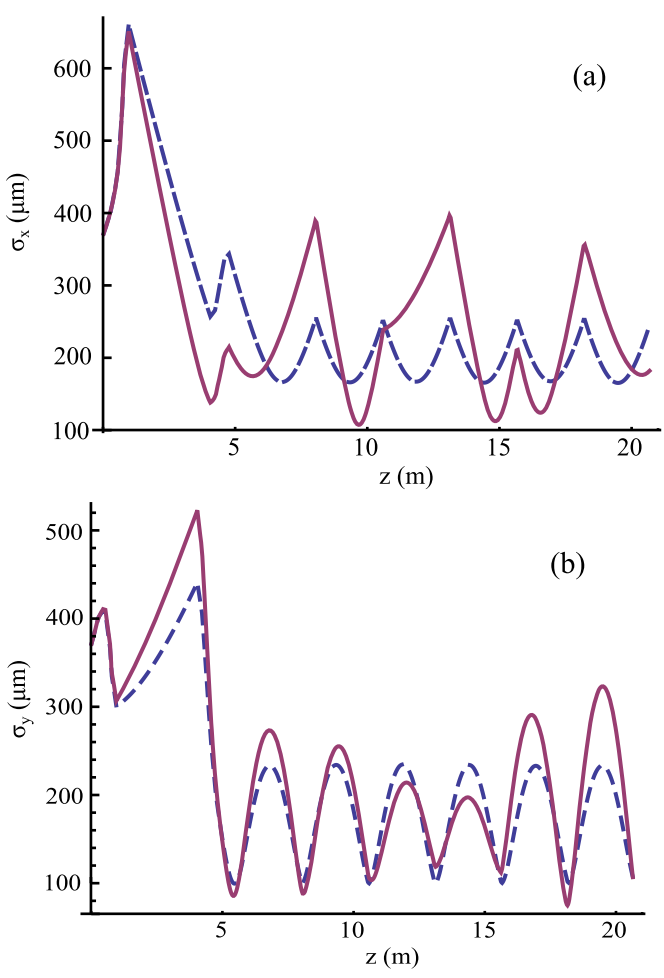

FIG. 2. Comparison between the matching (blue dashed line) and an alternative transport (red continuous line) condition for the e-beam from the linac exit to the end of the undulator with the beam parameters given in Table I. The behavior of the two transverse beam sizes $\sigma_{x}$ and $\sigma_{y}$ is reported respectively in (a) and (b). 


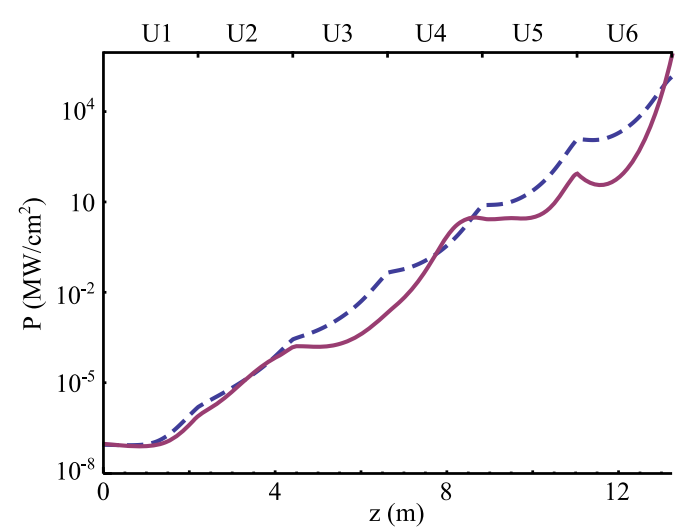

FIG. 3. An example of FEL intensity evolution along the undulator for the matched (blue dashed line) and transport (red continuous line) options in arbitrary units. The e-beam parameters at the linac exit are reported in Table I.

different matching and transport quadrupole configurations. The beam parameters for the simulation are detailed in Table I. From these results, it is furthermore clear that the canonical matching could not correspond to the maximum output power or to a shorter saturation length. Since the Pierce parameter is dependent on the current density, we expect that when the $\beta$ function decreases both a reduction of the gain length and an increase of the efficiency are produced.

Things are more complex for a real life SASE FEL device where the Pierce parameters are indeed affected by inhomogeneous broadening contributions associated with the gain decrease due to transverse and longitudinal phase space effects. Moreover, the diffraction effects play a further role in the reduction of the $\rho$ parameter. All these effects can be embodied in Eq. (1), without modifying the SLAP functional dependence, through a redefinition of the Pierce parameter, using a parametrization such as the one suggested in Refs. $[10,11,14]$. By keeping all these effects in the SLAP it is possible to get a sufficiently accurate description of the experiment, which can be exploited in real time to adjust e.g., the quadrupole currents, thus changing the matching conditions to drive the FEL output intensity towards an optimization.

TABLE I. Electron beam parameters for the simulation. The Twiss parameters are given at the linac exit.

\begin{tabular}{lc}
\hline \hline Energy $E$ & $90.95 \mathrm{MeV}$ \\
Relative energy spread $\sigma_{E}$ & $3.3 \times 10^{-3}$ \\
Normalized emittance $\epsilon_{x}$ & $5.28 \mathrm{~mm} \mathrm{mrad}$ \\
Normalized emittance $\epsilon_{y}$ & $4.38 \mathrm{~mm} \mathrm{mrad}$ \\
Twiss $\beta_{x}$ & $11.35 \mathrm{~m}$ \\
Twiss $\beta_{y}$ & $33.80 \mathrm{~m}$ \\
Twiss $\alpha_{x}$ & 0.81 \\
Twiss $\alpha_{y}$ & -2.06 \\
Bunch charge $Q$ & $160 \mathrm{pC}$ \\
rms bunch length $\Delta t$ & $1.69 \mathrm{ps}$ \\
\hline \hline
\end{tabular}

\section{SOFTWARE IMPLEMENTATION}

A graphic user interface (GUI) has been developed, in the Lab-view software environment, in order to implement experimentally the transport conditions, specified to optimize e.g., the output power. Using the experimental data (transversal beam emittances and Twiss parameters, energy, current profile and longitudinal phase space properties) given from the electron beam characterization and read by GUI interactively from the SPARC control system, an algorithm written in $\mathrm{C}++$ programming language provides the desired output.

A first subroutine calculates the quadrupole current relative to the single element of the FODO lattice composed by undulator-drift-quadrupole-drift by setting the matching conditions (namely by fixing the periodicity condition of the Twiss parameter at the entrance and exit of the lattice element). In the second step, the currents of the six quadrupoles along the transfer line are specified in order to match the beam Twiss parameter at the linac exit with those at the undulator entrance.

A successive subroutine generates, for each quadrupole, normal distributed random currents with an average value equal to the matching current and with a standard deviation equal to $0.03 \mathrm{~A}$. By using the SLAP procedure, the final FEL density power is calculated for the different settings of the quads current in the random distribution. The chosen values are those which maximize the output power. The GUI allows one to visualize the evolution of the FEL power along the six undulators.

The electron beam spot evolution along the six undulators is also simulated and visualized in the GUI panel at the position of the spot monitors located just after each undulator section of the SPARC FEL line.

\section{PRELIMINARY EXPERIMENTAL RESULTS}

The algorithm and the described procedure have been preliminarily checked on the SPARC FEL facility $[19,20]$.

TABLE II. Experimental quadrupoles' current settings for the two different matching and transport conditions. The positions of the quadrupoles are those shown in Fig. 1.

\begin{tabular}{lcc}
\hline \hline Quadrupole & Matching current $(\mathrm{A})$ & Transport current $(\mathrm{A})$ \\
\hline Q1 & 1.256 & 1.278 \\
Q2 & 0 & 0 \\
Q3 & -1.562 & -1.576 \\
Q4 & -0.727 & 0.741 \\
Q5 & 0 & 0 \\
Q6 & 1.653 & 1.735 \\
Q7 & 1.551 & 1.524 \\
Q8 & 1.556 & 1.485 \\
Q9 & 1.612 & 1.502 \\
Q10 & 1.494 & 1.558 \\
Q11 & 1.570 & 1.644 \\
\hline
\end{tabular}




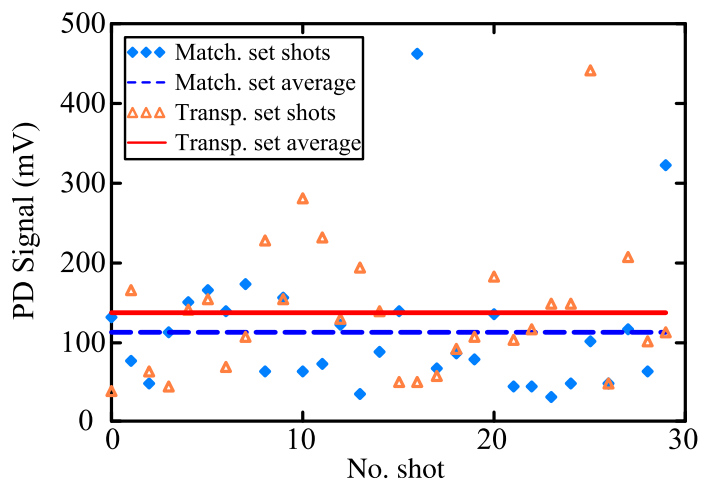

FIG. 4. Experimental sets of data corresponding to the different matching and transport configuration for the magnetic elements in the SPARC FEL (Table II). The signal detected by the photodiode (PD) is pointed together with the average value for the two sets of data. Only a relative difference can be appreciated in intensity since the PD is not calibrated; the beam parameters are reported in Table III.

Both the matching and the transport configurations have been one by one explored experimentally, using the GUI interface, to drive the electron beam into the undulator tuned at a resonance wavelength $\lambda_{0}=800 \mathrm{~nm}$. The quadrupoles' settings found for the two configurations are reported in Table II. A photodiode has been used to measure the output power of the FEL. The power, obtained using the quadrupoles' currents generated by the matching conditions, has been compared with the one obtained according to the criteria discussed in this paper (Fig. 4). The expected power output variation, according to our program, was $+25 \%$ while we measured a variation of $+21 \%$ coming from the statistics of 30 shots acquired. The systematic error was in the order of $2 \%$.

In Fig. 5 we have reported the simulated intensity growth according to the already quoted options, both curves start

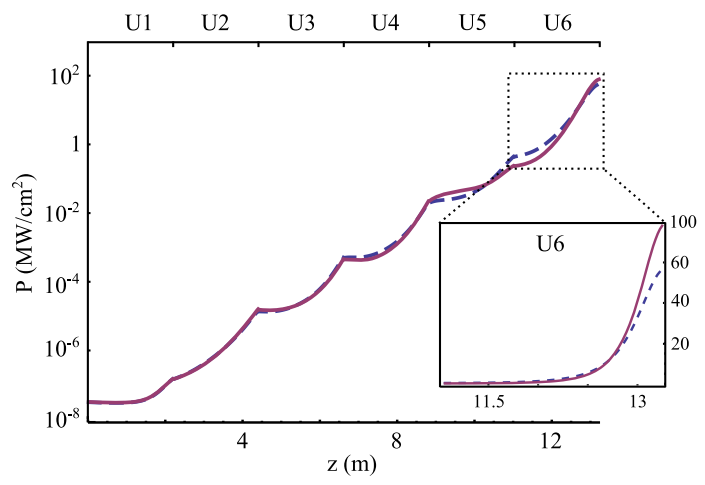

FIG. 5. Simulation of the FEL power growth evolution along the undulator for the matching (blue dashed line) and the transport (red continuous line) options, with the experimental conditions reported in Tables II and III. In the square a detail in linear scale of the evolution in the last undulator section is magnified.
TABLE III. Experimental condition of the beam at the linac exit.

\begin{tabular}{lc}
\hline \hline Energy $E$ & $88 \mathrm{MeV}$ \\
Relative energy spread $\sigma_{E}$ & $6.1 \times 10^{-3}$ \\
Normalized emittance $\epsilon_{x}$ & $4.75 \mathrm{~mm} \mathrm{mrad}$ \\
Normalized emittance $\epsilon_{y}$ & $3.17 \mathrm{~mm} \mathrm{mrad}$ \\
Twiss $\beta_{x}$ & $7.766 \mathrm{~m}$ \\
Twiss $\beta_{y}$ & $21.24 \mathrm{~m}$ \\
Twiss $\alpha_{x}$ & 0.98 \\
Twiss $\alpha_{y}$ & -1.228 \\
Bunch charge $Q$ & $160 \mathrm{pC}$ \\
rms bunch length $\Delta t$ & $0.707 \mathrm{ps}$ \\
\hline \hline
\end{tabular}

from the experimental parameters. The zoom on the last section is aimed to better appreciate (on a linear scale) the difference between the operating regimes.

\section{COMPARISON WITH GENESIS}

It can be argued that the SLAP procedure does not contain any phase information and therefore the results we have obtained are hampered by the fact that any change in the FEL dynamics is not correctly described and that the change in the focusing properties may induce dynamical effects associated with phase distortion, not properly accounted for in our parametrization.

To check the reliability of the method we have used a benchmark with GENESIS [6] which has been run using the same condition of the SLAP procedure. The comparison between the codes is reported in Fig. 6, where we show the power growth vs the undulator chain length.

In order to provide a meaningful comparison we configured the GENESIS magnetic lattice to be as similar as possible to the SPARC one. A good agreement between GENESIS and the SLAP has been observed for beam transversal sections up to about $0.06 \mathrm{~mm}^{2}$, while larger beam



FIG. 6. Comparison between GENESIS (blue dashed) and SLAP (amaranth continuous) for the whole undulator length. A detail, in linear scale, for the last undulator section is shown in the square. The length of the undulator takes into account also the drifts (steps in the graphic) between one undulator and the following one. Results are obtained using the e-beam parameters at the linac exit reported in Table IV. 


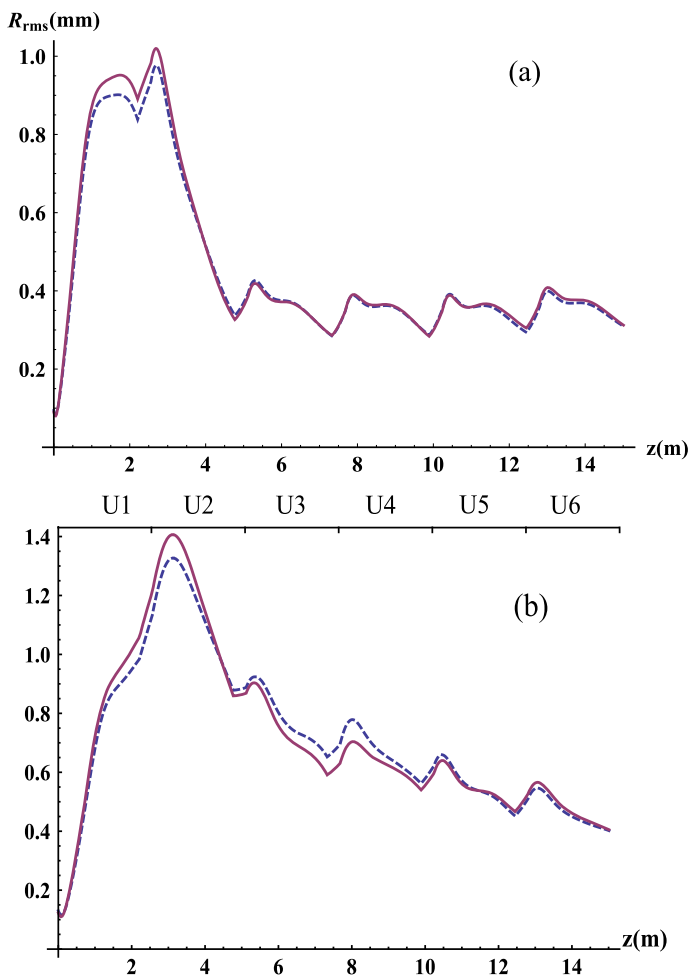

FIG. 7. Radiation spot size (rms) along the undulator calculated with GENESIS for the matching condition (blue dashed line) and an alternative transport (red continuous line). The cases (a) and (b) are relevant to an initial e-beam rms section of 0.06 and $0.1 \mathrm{~mm}^{2}$ respectively; a 450 A peak current and $160 \mathrm{pC}$ charge, the setting of the quadrupole currents are reported in Table V.

sizes result in a poorer agreement. This discrepancy can be ascribed to the diffractive effects, which are underestimated by SLAP and become increasingly important with the beam section. This effect is clearly shown (Fig. 7) where we

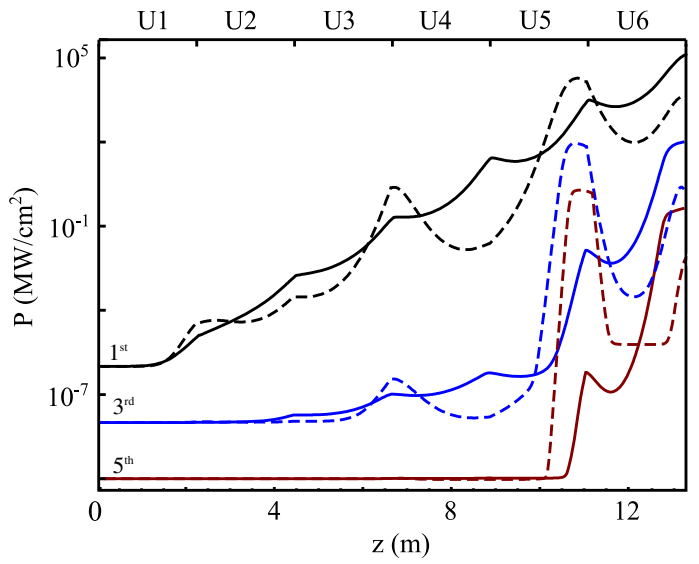

FIG. 8. Power evolution of the fundamental, third and fifth harmonics (line 1st, 3rd, 5th respectively) along the undulator. The simulation has been carried out for both the matching (solid line) and the transport option (dotted line). The e-beam parameters at the linac exit are reported in Table IV.
TABLE IV. Electron beam parameters used for the simulation. The Twiss parameters are given at the linac exit.

Energy $E$

Eenergy spread

Normalized emittance $\epsilon_{x}$

Normalized emittance $\epsilon_{y}$

Twiss $\beta_{x}$

Twiss $\beta_{y}$

Twiss $\alpha_{x}$

Twiss $\alpha_{y}$

Bunch charge $Q$

rms bunch length $\Delta t$

Beam peak current

$150 \mathrm{MeV}$

$0.408 \mathrm{MeV}$

$3.68 \mathrm{~mm} \mathrm{mrad}$

$3.68 \mathrm{~mm} \mathrm{mrad}$

$5.55 \mathrm{~m}$

$5.55 \mathrm{~m}$

$-1.736$

$-1.736$

$160 \mathrm{pC}$

$0.711 \mathrm{ps}$

$450 \mathrm{~A}$

TABLE V. Quadrupoles' current settings for two different conditions (matching and transport) used for GENESIS simulation reported in Fig. 7.

\begin{tabular}{lcc}
\hline \hline Quadrupole & Matching current (A) & Transport current (A) \\
\hline Q1 & -1.366 & -1.392 \\
Q2 & -3.739 & -3.62 \\
Q3 & 5.99 & 5.16 \\
Q4 & -4.349 & -4.36 \\
Q5 & -0.018 & -0.02 \\
Q6 & 3.691 & 3.76 \\
Q7 & 2.732 & 2.85 \\
Q8 & 2.741 & 2.78 \\
Q9 & 2.841 & 2.67 \\
Q10 & 2.632 & 2.66 \\
Q11 & 2.766 & 2.67 \\
\hline \hline
\end{tabular}

provide the radiation spot size evolution along the undulator for the SPARC transport line.

Before concluding this section it is worth stressing a further possibility offered by the control of the beam transport along the undulator line. As shown in Fig. 8 the condition to provide an enhancement of the nonlinear harmonic generation can be provided by an abrupt reduction of the transversal e-beam sections, which causes an early saturation inducing a fast blowup of the higher order harmonics.

\section{CONCLUSIONS}

In the previous sections we have discussed the application of the SLAP "protocol," conceived as an on-line benchmark for the SPARC experiment. A first, albeit preliminary, result of the procedure has been the check that a FEL SASE may safely operate even with a nonmatched transport line. The comparison done with more sophisticated codes yields substantial agreement with the results obtained with our analytical model. We underline that the present situation, albeit encouraging, is hampered by the SPARC operating conditions themselves, namely those of a test facility operating in the visible. A more 
consistent benchmark could be that achieved with different and more complex experimental configurations, provided by FEL operating at shorter wavelengths. A further element of uncertainty of the tool we propose is the rather naive inclusion of the beam transport elements in the analytical formulas describing the FEL parameters; even though the comparison with GENESIS is satisfactory, we believe that it cannot be considered conclusive. Within this respect a very encouraging result on the reliability of the protocol could be the check of the correctness of the predictions on the nonlinear harmonic generation reported in Fig. 8, which, we hope, will be experimentally verified in the near future.

[1] P. Emma et al., Nat. Photonics 4, 641 (2010).

[2] W. Ackermann et al., Nat. Photonics 1, 336 (2007).

[3] T. Shintake et al., Nat. Photonics 2, 555 (2008).

[4] M. Altarelli et al., DESY Report No. DESY-2006-097, 2006.

[5] G. Dattoli, M. Galli, and P. L. Ottaviani, ENEA Technical Report No. RT/INN/93/09, 1993.

[6] S. Reiche, Nucl. Instrum. Methods Phys. Res., Sect. A 429, 243 (1999).

[7] L. Giannessi, in Proceedings of the FEL Conference, 2006 (BESSY, Berlin, Germany, 2006).

[8] H. P. Freund, S. G. Biedron, and S. V. Milton, IEEE J. Quantum Electron. 36, 275 (2000).

[9] W. Fawley, Phys. Rev. ST Accel. Beams 5, 070701 (2002).
[10] M. Xie, in Proceedings of the Particle Accelerator Conference, Dallas, TX, 1995 (IEEE, New York, 1995).

[11] G. Dattoli, P. L. Ottaviani, and S. Pagnutti, Booklet for FEL Design: A Collection of Practical Formulae (ENEA Edizioni Scientifiche, Frascati, 2007).

[12] L. Giannessi et al., Phys. Rev. ST Accel. Beams 14, 060712 (2011).

[13] M. Artioli, G. Dattoli, P. L. Ottaviani, and P. Pagnutti, Virtual Laboratory and Computer Aided Design for Free Electron Lasers outline and simulation, Energia Ambiente e Innovazione n.3 2012, ENEA Press.

[14] M. V. Yurkov, E. V. Schneidmiller, and E. L. Saldin, The Physics of Free Electron Laser (Springer, New York, 2000).

[15] A. Zholents and M. Zolotorev, in Proccedings of the 4th Microbunching Instability Workshop, College Park, MD, 2012, pp. 55-60.

[16] G. Dattoli, E. Sabia, M. Del Franco, and A. Petralia, Nucl. Instrum. Methods Phys. Res., Sect. A 671, 82 (2012).

[17] G. Dattoli, E. Di Palma, A. Petralia, and M. Artioli, IEEE J. Quantum Electron. 49, 267 (2013).

[18] M. Quattromini, M. Artioli, E. Di Palma, A. Petralia, and L. Giannessi, Phys. Rev. ST Accel. Beams 15, 080704 (2012).

[19] M. Ferrario et al., Nucl. Instrum. Methods Phys. Res., Sect. B 309, 183 (2013).

[20] L. Giannessi et al., Phys. Rev. ST Accel. Beams 14, 060712 (2011). 